Notes and miscellanea

\section{HLA Antigens of the A and $B$ locus in relation to the development of silicosis}

Some years ago we studied the HLA antigens of the $A$ and $B$ locus in two groups of South African white gold miners with similar prolonged exposure to dust containing over $50 \%$ of free silica. Their various occupations throughout their working life were known, as were the relative dust exposures for the different occupations. It was therefore possible to compute a simple cumulative dust exposure index ("low dose equivalent years") for each man. This index is calculated by multiplying by two their time spent in "high dust" occupations and adding the years spent in "low dust" occupations.

Both groups of men had worked for at least 20 low dust equivalent years. One group of 45 had $H L A$ antigens in silicotic miners and two control groups radiological evidence of silicosis (category $1 / 0$ or higher, including only two with A shadows) and 56 had no evidence of silicosis.

Twenty nine HLA antigens were studied, and the antigen proportions in a control group of 279 Caucasian non-miners were available for comparison. The results are shown in the table.

There have been several studies ${ }^{1-4}$ of the relative proportions of HLA antigens of the A and $B$ locus in coal miners with and without coal workers' pneumoconiosis, but although differences (statistically significant by conventional methods) between coal workers with and without evidence of coal workers' pneumoconiosis have been found, no difference of significance has emerged when correction for the number of antigens tested for is carried out. In addition the different studies have not been consistent as to the antigen whose prevalence was suspected to be abnormal.

There has been only one previous study of these antigens in silicosis ${ }^{5}$ and a significant underrepresentation of antigen B7 was found in subjects with silicosis compared with non-silicotic silica exposed and non-exposed control groups $(\mathrm{p}=\mathbf{0 . 0 5}$ and $\mathrm{p}=$ 0.01 respectively).

In the present study the only finding was a

\begin{tabular}{|c|c|c|c|c|c|c|}
\hline \multirow[t]{2}{*}{279 Normal subjects } & \multicolumn{4}{|c|}{45 Silicotic miners } & \multicolumn{2}{|c|}{56 Non-silicotic miners } \\
\hline & & $\%$ & & $\%$ & & $\%$ \\
\hline $\begin{array}{l}1 \\
2 \\
3 \\
9(\text { W23 \& W24) } \\
11 \\
25 \\
26 \\
28 \\
29 \\
\text { W30 (with w31) } \\
\text { W32 } \\
\text { W34 }\end{array}$ & $\begin{array}{r}70 \\
133 \\
81 \\
72 \\
29 \\
9 \\
20 \\
26 \\
21 \\
25 \\
19 \\
4\end{array}$ & $\begin{array}{r}25 \cdot 1 \\
47 \cdot 7 \\
29 \cdot 0 \\
25 \cdot 8 \\
10 \cdot 4 \\
3 \cdot 2 \\
7 \cdot 2 \\
9 \cdot 3 \\
7 \cdot 5 \\
9 \cdot 0 \\
6 \cdot 8 \\
1 \cdot 4\end{array}$ & $\begin{array}{r}A \\
14 \\
19 \\
10 \\
8 \\
4 \\
3 \\
3 \\
7 \\
5 \\
3 \\
1 \\
0\end{array}$ & $\begin{array}{r}31 \cdot 1 \\
42 \cdot 2 \\
22 \cdot 2 \\
17 \cdot 8 \\
8 \cdot 9 \\
6 \cdot 7 \\
6 \cdot 7 \\
15 \cdot 6 \\
11 \cdot 1 \\
6 \cdot 7 \\
2 \cdot 2 \\
0\end{array}$ & $\begin{array}{r}12 \\
24 \\
16 \\
15 \\
4 \\
2 \\
0 \\
8 \\
5 \\
9 \\
5 \\
0\end{array}$ & $\begin{array}{r}21 \cdot 4 \\
42 \cdot 9 \\
28 \cdot 6 \\
26 \cdot 8 \\
7 \cdot 1 \\
3 \cdot 6 \\
0 \\
14 \cdot 3 \\
8 \cdot 9 \\
16 \cdot 1 \\
8 \cdot 9 \\
0\end{array}$ \\
\hline $\begin{array}{l}5 \\
7 \\
8 \\
12(W 44 \& W 45) \\
13 \\
14 \\
15 \\
17 \\
18 \\
27 \\
37 \\
40 \\
\text { W16 } \\
\text { W21 and W49 } \\
\text { W22 } \\
\text { W35 } \\
\text { W41 }\end{array}$ & $\begin{array}{l}31 \\
61 \\
47 \\
76 \\
14 \\
19 \\
42 \\
26 \\
24 \\
27 \\
7 \\
36 \\
27 \\
21 \\
14 \\
52 \\
1\end{array}$ & $\begin{array}{r}11 \cdot 1 \\
21.9 \\
16.8 \\
27.2(p=0.06) \\
5.0 \\
6.8 \\
15.1 \\
9.3 \\
8.6 \\
9.7 \\
2.5 \\
12.9(p=0.02) \\
9.7 \\
7.5 \\
5.0 \\
18.6 \\
0.4\end{array}$ & $\begin{array}{r}B \\
4 \\
10 \\
8 \\
7 \\
4 \\
6 \\
9 \\
6 \\
3 \\
4 \\
1 \\
1 \\
2 \\
3 \\
2 \\
8 \\
0\end{array}$ & $\begin{array}{r}8.9 \\
22.2 \\
17.8 \\
15.6 \\
8.9 \\
13.3 \\
20.0 \\
13.3 \\
6.7 \\
8.9 \\
2.2 \\
2.2 \\
4.4 \\
6.7 \\
4.4 \\
17.8\end{array}$ & $\begin{array}{r}5 \\
14 \\
9 \\
9 \\
3 \\
8 \\
7 \\
8 \\
4 \\
6 \\
2 \\
7 \\
3 \\
2 \\
2 \\
10 \\
0\end{array}$ & $\begin{array}{r}8 \cdot 9 \\
25 \cdot 0 \\
16 \cdot 1 \\
16 \cdot 1 \\
5 \cdot 4 \\
14 \cdot 3 \\
12 \cdot 5 \\
14 \cdot 3 \\
7 \cdot 1 \\
10 \cdot 7 \\
3 \cdot 6 \\
12 \cdot 5 \\
5 \cdot 4 \\
3 \cdot 6 \\
3 \cdot 6 \\
17 \cdot 9 \\
0\end{array}$ \\
\hline
\end{tabular}


decrease in B40 when compared with both silica exposed and non-exposed control groups whereas B7 was not abnormal.

G K SLUIS-CREMER Epidemiology Research Unit, Box 4584, Johannesburg, $S$ Africa.

G MAIER

Tissue Bank, South African Institute for Medical Research, Johannesburg, S Africa.

\section{References}

' Heise ER, Major PC, Mentnech MS, Parrish EJ, Jordan AL, Morgan WKC. Predominance of histocompatibility antigens W18 and HLA-A1 in miners resistant to complicated coal workers' pneumoconiosis. In: Walton WH, ed. Inhaled particles IV. Headington Hill Hall, Oxford: Pergamon Press, 1977.

${ }^{2}$ Heise ER, Mentnech MS, Olenchock SA, Kutz SA, Morgan WKC. HLA-A1 and coal workers' pneumoconiosis. Am Rev Respir Dis 1979;119:903-8.

${ }^{3}$ Wagner MMF, Darke C. HLA-A and B antigen frequencies in Welsh coalworkers with pneumoconiosis and Caplan's syndrome. Tissue Antigens 1979;14:165-8.

${ }^{4}$ Soutar CA, Coutts I, Parkes WR, et al. Histocompatibility antigens in coal miners with pneumoconiosis. Br J Ind Med 1983;40:34-8.

${ }^{5}$ Gualde N, De Leobardy J, Serizay B, Malinvaud G. HLA and silicosis. Am Rev Respir Dis 1977;166:334-6. 